\title{
Comportamentos de risco para a saúde de estudantes da Universidade Estadual do Norte do Paraná (Brasil) - uma proposta de intervenção online
}

\author{
Risk behavior for the health of students at the State University \\ of North Paraná, Brazil - a proposal for online intervention
}

\author{
Marcus Vinícius de Oliveira Mello ${ }^{1}$ \\ Rinaldo Bernardelli Junior ${ }^{1}$ \\ Berlis Ribeiro dos Santos Menossi ${ }^{1}$ \\ Fabio da Silva Ferreira Vieira ${ }^{1}$
}

${ }^{1}$ Centro de Ciências da Saúde, Universidade Estadual do Norte do Paraná. Al. Padre Magno 841 , Nova Jacarezinho. 86.400-000 Jacarezinho PR Brasil.

marcus_mello90@hotmail.com
Abstract Getting to know the lifestyles of university students provides important input for possible decision-making for the implementation of intervention programs within the university environment. The objective of this study was to analyze the prevalence of risk behaviors to health in a representative sample of UENP students and, in accordance with the information obtained, indicate specific actions using means that can assist in reducing such behaviors. The survey of the main risk factors for the health of the population in question was performed by database analysis. The orientation was conducted through social networks, with online control over access to and interactions with members of the networks using information made available by the research. More than 200 messages were posted, trying to put this population on the path towards factors related to healthy habits. A viewing average of $471.2 \pm 128.16$ people accessed the page and an average of 12,915.07 $\pm 6,517.45$ people saw the posts per week. Given the above, we suggest actions in UENP to encourage healthy habits, and the social networks proved to be a viable tool for this though other media may be offered, seeking to encourage this population to adopt a more active and health lifestyle.

Key words University students, Orientation, Social networking
Resumo Conhecer o estilo de vida de estudantes universitários torna-se relevante para subsidiar eventuais tomadas de decisões na implantação de programas intervencionistas no espaço da universidade. O objetivo deste trabalho foi analisar as prevalências de comportamentos de risco para a saúde em amostra representativa de estudantes da UENP e diante das informações levantadas, indicar ações específicas, através de meios que possam orientar na redução destes comportamentos. O levantamento dos principais fatores de risco para a saúde da população em questão foi feito por meio de análise de banco de dados. A orientação foi realizada através de redes sociais, com controle on line em relação aos acessos e interações dos membros das mesmas com as informações disponibilizadas por meio do trabalho. Foram realizados mais de 200 posts, buscando-se orientar esta população a fatores ligados a hábitos saudáveis. Atingiu-se uma visualização média de 471,2 $\pm 128,16$ pessoas por post e a página, um alcance médio de 12915,07 $\pm 6517,45$ pessoas por semana. Diante do exposto, sugerem-se ações na UENP, no sentido de orientação ligada a hábitos saudáveis $e$, as redes sociais mostraram-se como ferramenta viável para tal, porém outros meios podem ser ofertados, visando uma maior aderência desta população a um estilo de vida mais ativo e saudável.

Palavras-chave Universitários, Orientação, $R e$ des Sociais 


\section{Introdução}

O processo de urbanização, industrialização e desenvolvimento tecnológico ocorrido nas últimas décadas alterou profundamente a estrutura social das populações, principalmente daquelas residentes nos grandes centros urbanos. Isto influenciou decisivamente para que indivíduos de todas as idades passassem a adotar um estilo de vida cada vez menos saudável ${ }^{1}$.

A falta de atividade física associada a dietas inadequadas, tabagismo, consumo de bebidas alcoólicas e uso de outras drogas são determinantes na ocorrência e progressão das doenças crônicas não transmissíveis. De acordo com o Centers for Disease Control and Prevention ${ }^{2}$, a cada ano, mais de dois milhões de pessoas morrem em todo mundo, e no Brasil, as doenças crônico-degenerativas têm se apresentado como a primeira causa de morbimortalidade inclusive na população jovem ${ }^{3}$.

Em relação aos aspectos metabólicos ligados ao desenvolvimento de doenças, alguns fatores de risco são receptivos a alterações, ao contrário de determinadas condições metabólicas (doenças congênitas) que são praticamente inalteráveis ${ }^{4}$. Atividade física e o consumo de alimentos de maneira adequada protege o organismo de disfunções em seu funcionamento ${ }^{5}$. Desta forma, mudanças de comportamento antecipadas devem ser adotadas como medida preventiva.

Estudo do U.S. Department of Health and Human Services ${ }^{6}$ demonstrou alta prevalência de várias situações de risco nos hábitos de jovens universitários norte-americanos, como é o caso do tabagismo, do consumo de bebidas alcoólicas e de outras drogas, comportamentos sexuais indevidos, alimentação inadequada e sedentarismo. Além deste fator, hábitos nocivos à saúde na população universitária geralmente se associam ${ }^{7}$.

Há também outros comportamentos de risco à saúde, em especial à saúde mental, que podem ser encontrados no estudo de Bastos ${ }^{8}$, no qual é analisada a discriminação em inúmeras situações, entre elas a que estão sujeitos os que porventura façam o uso de drogas ou estejam fora dos padrões de estereótipos determinados pela sociedade atual, como por exemplo, os obesos.

A aquisição de conhecimentos e a tomada de ações positivas - alimentação equilibrada, prática de atividade física adequada e comportamentos preventivos - são consolidadas durante a juventude ${ }^{1}$.

Os jovens, cada vez mais, têm-se utilizado das redes sociais para aliviar as cargas da vida cotidia- $\mathrm{na}^{9}$, fazendo destas um exponencial meio de divulgação para esta população. No Ambiente das redes o compartilhamento de informação e de conhecimento entre as pessoas é constante, pois elas frequentemente gostam de compartilhar o que sabem ${ }^{10}$.

Caso pretenda-se que as pessoas de uma organização compartilhem o que aprenderam, devese criar condições para tal. A disposição em compartilhar de forma eficiente informações, entre os autores de uma rede, asseguram ganhos porque cada participante melhora, valendo-se delas, as quais passam a ter acesso e que poderão reduzir as incertezas e promover o crescimento mútuo ${ }^{11}$.

Explorar este tipo de ferramenta na busca por orientar universitários sobre hábitos de vida saudáveis vem a se tornar uma forma eficaz e acessível para que o conhecimento sobre tais informações cheguem até esta população, que é em sua maioria, jovem.

Diante do exposto, este trabalho tem por objetivo, analisar as prevalências de comportamentos de risco para a saúde em amostra representativa de estudantes da Universidade Estadual do Norte do Paraná e através deste levantamento, estruturar uma forma de orientação ligada aos principais fatores de risco.

\section{Métodos}

Para a elaboração do estudo foi utilizado o banco de dados construído a partir da tese de doutoramento em Ciências do Desporto do Prof. Dr. Rinaldo Bernardelli Junior, um levantamento descritivo de corte transversal, de base populacional, envolvendo informações relacionadas a uma seleção de indicadores sociodemográficos e comportamentos de risco para a saúde de universitários que frequentaram os cursos oferecidos pela Universidade Estadual do Norte do Paraná, Brasil (UENP).

A coleta dos dados foi realizada nos meses de abril a junho de 2008. Os protocolos de intervenção utilizados foram aprovados pelo Comitê de Ética em Pesquisa da Universidade Estadual Londrina e acompanharam normas da Resolução 196/96 ${ }^{12}$ do Conselho Nacional de Saúde sobre pesquisa envolvendo seres humanos.

\section{Caracterização da População}

A UENP é uma Instituição de ensino superior pública que ofereceu, no ano letivo de 2008, 22 cursos de graduação distribuídos em três campi: 
Campus Jacarezinho, abrigando o Centro de Ciências da Saúde, Centro de Letras Comunicação e Artes, Centro de Ciências Humanas e da Educação e Centro de Ciências Sociais e Aplicadas, contando com 2.539 alunos matriculados; Campus Luiz Meneghel de Bandeirantes, abrigando o Centro de Ciências Agrárias, Centro de Ciências Biológicas e Centro de Ciências Tecnológicas, contando com 1.660 alunos; Campus Cornélio Procópio, que abriga o Centro de Ciências Sociais Aplicadas, Centro de Letras Comunicação e Artes e Centro de Ciências Humanas e da Educação, contando com 1.882 alunos matriculados. Os três campi da UENP estão localizados na mesorregião Norte Pioneiro do Estado do Paraná, no sul do Brasil, a qual abrange 46 municípios, perfazendo um total de aproximadamente 540 mil habitantes. A população alvo selecionada para o presente estudo foram os universitários matriculados nos três campi da UENP.

\section{Pesquisa de campo}

A coleta dos dados foi realizada mediante instrumento de medida constituído de duas seções: indicadores sociodemográficos e comportamentos de risco para saúde. Na seção relacionada aos indicadores sociodemográficos foram levantadas informações quanto ao gênero, à idade, à etnia, ao estado conjugal, ao curso, ano e período que estuda, ao núcleo familiar, ao nível de escolaridade dos pais, à realização de trabalho remunerado, ao plano de família e à classe econômica da família.

As informações associadas aos comportamentos de risco para a saúde foram obtidas mediante a aplicação do questionário Youth Risk Behavior Survey - College (YRBS-C), traduzido para o idioma português e adaptado transculturalmente pelo grupo de pesquisa coordenado pelo Prof. Dr. Dartagnan Pinto Guedes, da Universidade Estadual de Londrina (UEL). Neste caso, o YRBS-C caracteriza-se por questionário que procura reunir informações referentes aos comportamentos de risco para a saúde durante a formação universitária, dividido em grupos de questões relacionadas: (a) à segurança pessoal; (b) à violência; (c) à intenção de suicídio; (d) ao uso de tabaco; (e) ao consumo de bebidas alcoólicas; (f) ao uso de maconha; (g) ao uso de outras drogas; (h) à relação sexual; (i) ao controle do peso corporal; (j) à alimentação; e (1) à prática de atividade física.

$\mathrm{O}$ instrumento de medida com as duas seções foi aplicado em um único momento, individualmente para cada universitário, por um úni- co pesquisador e no próprio local e horário das aulas. Para tanto, as salas de aulas foram visitadas e aqueles universitários que concordaram em participar do estudo receberam orientações quanto ao preenchimento do Termo de Consentimento Livre e Esclarecido. Na sequencia, os participantes do estudo receberam o instrumento com instruções e recomendações para o seu preenchimento, não sendo estabelecido limite de tempo para o seu término. As eventuais dúvidas manifestadas pelos respondentes foram prontamente esclarecidas pelo pesquisador que acompanhava a coleta dos dados.

\section{Seleção da Amostra}

De um universo de 6.081 universitários, 3.356 $(55,2 \%)$ sujeitos, 1.988 do gênero feminino e 1.368 do gênero masculino, concordaram voluntariamente em participar do estudo.

Os critérios adotados para exclusão de algum sujeito selecionado para o estudo foram à recusa do preenchimento do Termo de Consentimento Livre e Esclarecido e quando os questionários possuíam quantidades elevadas de questões não respondidas ou contendo respostas inválidas ou absurdas.

Dessa forma, foram excluídos 52 sujeitos, sendo 29 do gênero feminino e 23 do gênero masculino. Portanto, a amostra definitiva para o estudo foi composta por 1.959 e 1.345 respectivamente, totalizando 3.304 sujeitos.

\section{Análise dos dados \\ e aplicação de programas preventivos}

Após análise dos dados, buscando-se estabelecer os principais fatores de risco para a saúde dos universitários da UENP, foram realizadas postagens em redes sociais, objetivando, por meio destas, orientar esta população a fatores ligados ao consumo de drogas lícitas e ilícitas, atividade sexual segura, hábitos alimentares saudáveis e de prática de atividade física adequada, os quais se espera que possam contribuir para a redução dos comportamentos de risco bem como a adoção de um estilo de vida saudável.

Para tal, foram criados um perfil no twitter $^{\circledR}$ e uma página no facebook ${ }^{\circledR}$, titulados "Ser Saúde - UENP" que foram divulgados no site oficial da UENP, em grupos ativos dos alunos da instituição nas redes, foram também confeccionados 3000 flyers e 40 cartazes, os quais foram distribuídos e fixados em todos os blocos de todos os centros dos Campi da UENP. 


\section{Análise Estatística}

Os dados foram analisados utilizando-se o pacote estatístico computadorizado Statistical Package for the Social Science (SPSS), versão 17.0. As taxas de prevalências relacionadas aos comportamentos de risco para a saúde foram estimadas a partir de alternativas de respostas de cada questionamento do instrumento, acompanhadas pelos respectivos intervalos de confiança a 95\%. Eventuais diferenças significativas entre ambos os gêneros foram analisadas mediante aplicação do teste de qui-quadrado. Após analisados os principais fatores de risco para a saúde foram criados os meios de orientação pelas redes sociais, onde foi feito controle on line em relação aos acessos e interações dos membros das redes com as informações disponibilizadas por meio do trabalho semanalmente, possibilitando ao final deste, informar o alcance que as mesmas obtiveram.

\section{Resultados}

A página no facebook $^{\circledR}$ atingiu uma visualização média de $12.915,07 \pm 6.517,45$ pessoas por semana e o perfil no twitter ${ }^{\circledR}$ conta com mais de 400 seguidores. Em relação ao alcance, foram atingidos cerca de 40 municípios, dos quais, mais de 25 são localizados na mesorregião no norte pioneiro do Paraná. Também foram alcançadas pessoas em outros países, tais como Argentina, Bolívia, Paraguai, Portugal, Espanha, Canadá e Estados Unidos. A Tabela 1 apresenta o perfil geral das pessoas que acessaram a página, em relação a gênero e faixa etária.

Em relação às postagens, foram realizadas mais de 200, buscando-se orientar sobre:

1 - prática de atividade física: abordando atividades físicas e exercícios aeróbios, anaeróbios e sobre seus benefícios, assim como incentivando os seguidores a adotarem um estilo de vida fisicamente mais ativo e saudável.
2 - hábitos alimentares: salientando sobre alimentos saudáveis bem como a importância no funcionamento de determinados órgãos de acordo com o tipo de alimento, benefícios da ingestão adequada destes, assim como, informando sobre alimentos que podem ser prejudiciais ao organismo.

3 - drogas: foram realizados posts relacionados a drogas lícitas e ilícitas, alertando sobre os efeitos deletérios destas no organismo humano.

4 - doenças: foram abordadas doenças cardiorrespiratórias, neurológicas, reumatológicas e endócrinas, alertando sobre sinais, sintomas, causas e consequências, e também sobre como preveni-las e evitá-las.

5 - atividade sexual: realizados posts no sentido de orientação da prática de atividade sexual segura e alertando sobre doenças sexualmente transmissíveis, assim como, informando sobre os benefícios da prática sexual, principalmente quando feita com segurança.

6 - segurança no trânsito: realizados posts tratando do assunto, ressaltando a importância da utilização de equipamentos de segurança (cinto de segurança, capacete, etc.) e cuidados gerais que devem ser tomados no trânsito de acordo com recomendações dos órgãos competentes.

Os resultados obtidos e quantidade de posts de acordo com cada tema são visualizados pela Tabela 2.

Em relação ao alcance geral da página, foram expostos aqui o alcance das três (03) semanas anteriores ao fechamento dos dados para submissão do trabalho. A Tabela 3 apresenta o alcance geral da página, incluindo a "quantidade de fãs" tratando das pessoas que recebem de forma direta o que é postado; os "amigos de fãs", que recebem de forma indireta os assuntos; a quantidade de "pessoas falando sobre", relacionado às que estão interagindo com o conteúdo, compartilhando tais informações; e, o "alcance total na semana" informando a quantidade de pessoas que a página atingiu na semana em questão.

Tabela 1. Alcance em relação ao gênero e faixa etária.

\begin{tabular}{lccccrr}
\hline \multirow{2}{*}{ Gênero } & \multicolumn{5}{c}{ Faixa etária } \\
\cline { 2 - 6 } & $\mathbf{1 3 - 1 7}$ anos & $\mathbf{1 8 - 2 4}$ anos & $\mathbf{2 5 - 3 4}$ anos & $\mathbf{3 5 - 4 4}$ anos & $\mathbf{4 5 - 5 4}$ anos & $\mathbf{5 5}$ ou + \\
\hline Feminino (55,71\%) & $9 \%$ & $30 \%$ & $11 \%$ & $3,1 \%$ & $1,9 \%$ & $0,71 \%$ \\
Masculino (44,29\%) & $5,9 \%$ & $24 \%$ & $9,9 \%$ & $1,9 \%$ & $1,8 \%$ & $0,79 \%$ \\
Total (100\%) & $14,9 \%$ & $54 \%$ & $20,9 \%$ & $5 \%$ & $3,7 \%$ & $1,5 \%$ \\
\hline
\end{tabular}

Fonte: Própria. 


\section{Discussão}

A ferramenta utilizada foi lançada em março de 2012, aproximadamente 1700 pessoas a apoiaram diretamente e esta obteve um alcance médio de $12.915,07 \pm 6.517,45$ pessoas por semana.

Observando-se os resultados de uma forma geral, nota-se um maior acesso de pessoas do gênero feminino $(55,71 \%)$, o que vem ao encontro do número de voluntários participantes do levantamento de dados, onde a amostra foi composta, em sua maioria, por mulheres (59,29\%). Esta maior participação justifica-se com base nas denominadas Razões de Gênero, que expressam a quantidade de habitantes de homens para cada grupo de 100 mulheres em determinado grupo populacional. Caso seu escore seja inferior a 100, significa que no universo estudado o contingente feminino supera o masculino ${ }^{13}$. De acordo com os dados do senso de 2010, a população brasileira é composta, aproximadamente, $51 \%$ por mulheres ${ }^{14}$.

Em relação às cidades alcançadas, a página atingiu mais de $55 \%$ dos 46 municípios da mesorregião do norte pioneiro do Paraná. Uma das características marcantes da UENP é que a maioria dos estudantes é oriunda de municípios não muito distantes das sedes dos três (3) campus, o que oportuniza residirem com os seus familiares, à exceção de alguns cursos de período inte-

Tabela 2. Alcance em relação aos posts realizados.

\begin{tabular}{lcc}
\hline \multicolumn{1}{c}{ Tema } & $\begin{array}{c}\text { Quantidade } \\
\text { de } \text { posts }\end{array}$ & Alcance \\
\hline Atividade Física & 74 & $426,55 \pm 104,09$ \\
Hábitos Alimentares & 80 & $615,5 \pm 42,96$ \\
Drogas & 22 & $423 \pm 180,5$ \\
Doenças & 14 & $472 \pm 128,18$ \\
Atividade Sexual & 26 & $489,66 \pm 137,38$ \\
Segurança no Trânsito & 11 & $503,5 \pm 70,7$ \\
Total & 227 & $471,2 \pm 128,16$ \\
& &
\end{tabular}

Fonte: Própria. gral, que dificultam o retorno dos estudantes para suas cidades, mesmo que próximas ${ }^{1}$. Sendo assim, as cidades alcançadas foram as quais residem grande parte dos universitários da UENP.

Quanto à faixa etária, ocorreu um maior alcance de pessoas entre 18 e 24 anos (54\%). Os voluntários participantes do levantamento de dados tinham, em sua maioria, também entre 18 e 24 anos (44,6\%), o que corrobora com dados da educação brasileira em relação à faixa etária da população universitária ${ }^{1}$.

\section{Conclusões}

A rede mundial de computadores é sem dúvida, uma forma eficiente e eficaz de se atingir um grande número de pessoas de maneira rápida e barata, haja vista as manifestações que ocorrem mundo afora, que são planejadas e divulgadas pela Internet. Há infinitas páginas com objetivos dos mais diversos, entre elas há as que tratam de divulgar orientações sobre saúde, o que sugere que esta seja uma ferramenta exequível para divulgação de propostas.

Analisando-se os locais onde a ferramenta foi divulgada, lembrando que estes foram: site oficial da UENP; grupos ativos de alunos da instituição nas redes; além de flyers e cartazes distribuídos em todos os centros de todos os Campi da UENP, e, de acordo com a faixa etária e a localidade das pessoas que acessaram as informações disponibilizadas por meio do trabalho, prospecta-se que a população alvo foi atingida.

Sendo assim, sugerem-se ações na UENP, no sentido de orientação ligada a hábitos saudáveis e as redes sociais mostraram-se como ferramenta viável para tal, porém outros meios podem ser ofertados, visando uma maior aderência desta população a um estilo de vida mais ativo e saudável.

Tabela 3. Visualização geral da página na $16^{\mathrm{a}}$, $17^{\mathrm{a}}$ e $18^{\mathrm{a}}$ semana após o lançamento.

\begin{tabular}{lcccc}
\hline & Quantidade de fãs & Amigos de fãs & Pessoas falando sobre & Alcance total na semana \\
\hline 16a semana & 1.394 & 389.559 & 186 & 12.055 \\
$17^{\text {a }}$ semana & 1.461 & 398.307 & 421 & 24.130 \\
$18^{\text {a }}$ semana & 1.508 & 418.460 & 167 & 14.119 \\
\hline
\end{tabular}

Fonte: Própria. 


\section{Colaboradores}

MVO Mello e R Bernardelli Júnior participaram da concepção, delineamento, análise e interpretação dos dados, redação do artigo, aprovação da versão a ser publicada; BRS Menossi da concepção, delineamento, análise e interpretação dos dados; e, FSF Vieira da interpretação dos dados, redação do artigo.

\section{Referências}

1. Bernardelli Júnior R. Comportamentos de Risco para a Saúde de Estudantes da Universidade Estadual do Norte do Paraná, Brasil [tese]. Vila Real: Universidade de Trás-os-Montes e Alto Douro; 2010.

2. Centers for Disease Control and Prevention (CDC). Non fatal and sports - recreation-related injuries treated in emergency departments - United States, July 2000-July 2001. MMWR Morb Mortal Wkly Rep. 2002; 51(3):736-740.

3. Cotrin CB, Carvalho GC, Gouveia N. Comportamento de saúde entre jovens estudantes das redes pública e privada de área metropolitana de São Paulo. Rev Saude Publica 2000; 34(6):636-645.

4. Malina RM, Bouchard CG. Maturation and physical activity. Chanpaign: Human Kinetics; 1991.

5. Castanho F, Marsola FC, McLellan KCP, Nicola M, Moreto F, Burini RC. Consumo de frutas, verduras e legumes associado à Síndrome Metabólica e seus componentes em amostra populacional adulta. Cien Saude Colet 2013; 18(2):385-392.

6. Department of Health and Human Services (USDHHS). Measuring Healthy Days. Atlanta: Center for Disease Control and Prevention; 2000. [página na Internet]. [acessado 2012 mar 31]. Disponível em: http://www.cdc.gov/nccdphp/brfss/htm.

7. Granville-Garcia AF, Sarmento DJS, Santos JA, Pinto TA, Sousa RV, Cavalcanti AL. Smoking among undergraduate students in the area of health. Cien Saude Colet 2012; 17(2):389-396.

8. Bastos JLD. Desigualdades "raciais” em saúde: Medindo a experiência de discriminação auto-relatada no Brasil 2010 [tese]. Pelotas: Universidade Federal de Pelotas; 2010.

9. Andrade GRB, Vaitsman J. Apoio Social e Redes: Conectando Solidariedade e Saúde. Cien Saude Colet 2002; 7(4):925-934,

10. Tomaél MI, Marteleto RM. Redes Sociais: posições dos atores no fluxo da informação. Bibliotecon. Ci. Inf. Florianópolis, 2006. [Periódico na Internet] 2006. [acessado 2012 abr 22]. Disponível em: http:// redalyc.uaemex.mx/redalyc/pdf/147/14720365008. pdf

11. Dixon NM. Common knowledge: how companies thrive by sharing what they knowledge. Boston: Harvard Business School Press; 2000.

12. Brasil. Ministério da Saúde (MS). Conselho Nacional de Saúde. Resolução no. 196 de 10 de outubro de 1996. Diretrizes e Normas Regulamentadoras de Pesquisas Envolvendo Seres Humanos. Diário Oficial da União 1996; 16 out.

13. Oliveira JC, Pereira NOM. População Jovem no Brasil: A Dimensão Demográfica. Rio de Janeiro: IBGE; 1999.

14. Instituto Brasileiro de Geografia e Estatística (IBGE). Pesquisa Nacional por Amostra de Domicílios - PNAD. [banco de dados na Internet] 2010. [acessado 2012 abr 22]. Disponível em: http://www.ibge. gov.br/ home/estatistica/pesquisas/pesquisa_resultados. php?id_pesquisa $=40$

Artigo apresentado em 26/10/2012

Aprovado em 30/07/2013

Versão final apresentada em 20/08/2013 